\title{
TWO WEIGHTS WITH ORTHOGONAL SUPPORTS BUT EQUAL ON A DENSE *-SUBALGEBRA
}

\author{
BY A. VAN DAELE
}

\begin{abstract}
We construct two normal semifinite weights $\varphi$ and $\psi$ on $B(H)$ with orthogonal supports and such that $\varphi(x)=\psi(x)$ for $x$ in a weakly dense ${ }^{*}$-subalgebra $M_{0}$ contained in $\mathbb{M}_{\varphi}$ and $\mathbb{R}_{\psi}$. The example is based on the existence of a pair of positive self-adjoint operators $h$ and $k$ on $H$ with orthogonal supports and such that $\|h \xi\|=\|k \xi\|$ for $\xi$ in a dense subspace $D_{0}$ contained in $D(h) \cap D(k)$.

By a slight modification we obtain two commuting faithful normal semifinite weights on $B(H)$ that agree on a weakly dense *-subalgebra. This shows that the condition of invariance of this subalgebra for the modular automorphism group may not be omitted in the theorem of Pedersen and Takesaki on equality of weights [2, Proposition 5.9].
\end{abstract}

Let $M$ be a von Neumann algebra. If $\varphi$ is a normal semifinite weight on $M$ denote $\mathfrak{N}_{\varphi}=\left\{x \in M \mid \varphi\left(x^{*} x\right)<\infty\right\}$ and $\mathfrak{M}_{\varphi}=\mathfrak{N}_{\varphi}^{*} \mathfrak{N}_{\varphi}$. It is well known in the theory of weights that $\mathfrak{N}_{\varphi}$ is a left ideal and that $\mathfrak{M}_{\varphi}$ is a ${ }^{*}$-subalgebra spanned by its positive part $\mathfrak{M}_{\varphi}^{+}$which is equal to $\left\{x \in M^{+} \mid \varphi(x)<\infty\right\}$. The weight has a unique extension, which we still denote by $\varphi$, to a linear functional on $\mathfrak{M l}_{\varphi}$. Because $\varphi$ is assumed to be semifinite, the subalgebra $\mathfrak{M}_{\varphi}$ is weakly dense.

We will only be concerned with weights on the von Neumann algebra $B(H)$ of all bounded linear operators on a Hilbert space $H$. In the next proposition we will use the notation $\xi \otimes \eta$ for the rank one operator on $H$ defined by $(\xi \otimes \eta) \zeta=$ $\langle\zeta, \eta\rangle \xi$ whenever $\xi, \eta, \zeta \in H$.

1. Proposition. There is a one-to-one correspondence between the set of positive selfadjoint operators $h$ on $H$ and the set of normal semifinite weights $\varphi$ on $B(H)$ given by $\varphi(\xi \otimes \xi)=\|h \xi\|^{2}$ if $\xi \in D(h)$ and $\varphi(\xi \otimes \xi)=\infty$ if $\xi \notin D(h)$.

This result essentially follows from the work of Pedersen and Takesaki [2] but can also be proved directly using a technique as in Lemma 1.4 of [1]. Moreover we have that $\varphi$ is faithful if and only if $h$ is nonsingular, and in that case the modular automorphisms are given by $\sigma_{t}(x)=h^{2 i t} x h^{-2 i t}$ for all $x \in B(H)$ and all $t \in \mathbf{R}$.

To prove our main result we need a pair of positive selfadjoint operators with certain properties. The existence of such a pair was shown already in [3]. The proof is short and simple and we also give it here for completeness.

Received by the editors April 2, 1981.

1980 Mathematics Subject Classification. Primary 46L10, 46L50.

() 1981 American Mathematical Society 0002-9904/81/0000-0405/\$01.75 
2. Proposition. There exists a pair of positive selfadjoint operators $h$ and $k$ with orthogonal supports and such that $\|h \xi\|=\|k \xi\|$ for $\xi$ in a dense subspace $D_{0}$ contained in $D(h) \cap D(k)$.

Proof. Let $a$ and $b$ be two selfadjoint operators on a Hilbert space $H_{0}$ such that $O(a) \cap D(b)=\{0\}$. If we replace $a$ and $b$ by $a^{*} a+1$ and $b^{*} b+1$ we may assume, if necessary, that $a$ and $b$ are positive with bounded inverses satisfying $0 \leqslant a^{-1} \leqslant 1$ and $0 \leqslant b^{-1} \leqslant 1$.

Consider $H=H_{0} \oplus H_{0}$ and define the operators $h$ and $k$ on $H$ by

$$
\begin{aligned}
& D(h)=\left\{(\xi, \eta) \mid \xi \in D(a), \eta \in H_{0}\right\}, \\
& D(k)=\left\{(\xi, \eta) \mid \xi \in H_{0}, \eta \in D(b)\right\}
\end{aligned}
$$

and $h(\xi, \eta)=(a \xi, 0)$ if $(\xi, \eta) \in D(h)$ and $k(\xi, \eta)=(0, b \eta)$ if $(\xi, \eta) \in D(k)$. Then $h$ and $k$ are positive selfadjoint operators with orthogonal supports.

If we also let $D_{0}=\left\{\left(a^{-1} \eta, b^{-1} \eta\right) \mid \eta \in H_{0}\right\}$ then $D_{0} \subseteq D(h) \cap D(k)$ and $\|h \xi\|=\|k \xi\|$ for all $\xi \in D_{0}$. To prove that $D_{0}$ is dense we assume that $\left(\eta_{1}, \eta_{2}\right)$ is a pair in $H$ such that $\left(\eta_{1}, \eta_{2}\right) \perp\left(a^{-1} \eta, b^{-1} \eta\right)$ for all $\eta \in H_{0}$. Then $\left\langle a^{-1} \eta_{1}+b^{-1} \eta_{2}, \eta\right\rangle=\left\langle\eta_{1}, a^{-1} \eta\right\rangle+\left\langle\eta_{2}, b^{-1} \eta\right\rangle=0$ for all $\eta \in H_{0}$ so that $a^{-1} \eta_{1}=-b^{-1} \eta_{2}$. Because $D(a) \cap D(b)=\{0\}$ it follows that $a^{-1} \eta_{1}=b^{-1} \eta_{2}$ $=0$ and $n_{1}=n_{2}=0$. This completes the proof.

It is now easy to obtain our main result.

3. THEOREM. There exists a pair of normal semifinite weights $\varphi$ and $\psi$ on $B(H)$ with orthogonal supports such that $\varphi(x)=\psi(x)$ for $x$ in a weakly dense *-subalgebra $M_{0}$ of $B(H)$ contained in $\mathfrak{M}_{\varphi}$ and $\mathfrak{M}_{\psi}$.

Proof. Choose a pair of positive selfadjoint operators $h$ and $k$ on $H$ with orthogonal supports but such that $\|h \xi\|=\|k \xi\|$ for all $\xi$ in a dense subspace $D_{0}$ contained in $D(h) \cap D(k)$. Let $\varphi$ and $\psi$ be the corresponding weights on $B(H)$.

If $e$ is the support projection of $h$ then $(1-e) H \subseteq D(h)$ and $h(1-e)=0$. And if $\left\{\xi_{\alpha} \mid \alpha \in A\right\}$ is an orthonormal basis for $H$ we get

$$
\varphi(1-e)=\sum_{\alpha \in A} \varphi\left((1-e) \xi_{\alpha} \otimes(1-e) \xi_{\alpha}\right)=\sum_{\alpha \in A}\left\|h(1-e) \xi_{\alpha}\right\|^{2}=0
$$

so that the support of $\varphi$ is contained in $e$. Therefore $\varphi$ and $\psi$ will have orthogonal supports. Now let $M_{0}$ be the algebra of linear combinations of the operators $\left\{\xi \otimes \eta \mid \xi, \eta \in D_{0}\right\}$. If $\xi \in D_{0}$ then $\varphi(\xi \otimes \xi)=\|h \xi\|^{2}=\|k \xi\|^{2}=\psi(\xi \otimes \xi)$ and by linearity and some kind of polarization we obtain $\varphi(x)=\psi(x)$ for all $x \in M_{0}$. And finally because $D_{0}$ is dense in $H$ also $M_{0}$ will be norm dense in the algebra of finite rank operators, and so weakly dense in $B(H)$. 
Pedersen and Takesaki have considered the problem of equality of two normal semifinite weights when they coincide on a dense *-subalgebra. They showed that $\varphi$ and $\psi$ are equal if they commute (i.e. if $\psi$ is invariant for the modular automorphisms of $\varphi$ ) and if they agree on a dense ${ }^{*}$-subalgebra which is invariant under the modular automorphisms of $\varphi$. Our next result shows that the invariance of the subalgebra cannot be omitted, and only a small modification to the example in Theorem 3 has to be made.

4. THEOREM. There exists a pair of different faithful normal semifinite weights $\varphi$ and $\psi$ on $B(H)$ such that $\psi$ is invariant for the modular automorphisms

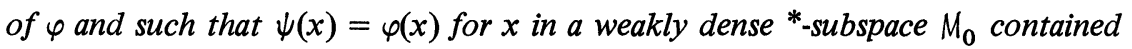
in $\mathfrak{M}_{\varphi} \cap \mathfrak{M}_{\psi}$.

Proof. Again let $h$ and $k$ be as in Proposition 2. Then consider $h_{1}=$ $\left(h^{2}+1\right)^{1 / 2}$ and $k_{1}=\left(k^{2}+1\right)^{1 / 2}$ and let $\varphi$ and $\psi$ be the weights associated to $h_{1}$ and $k_{1}$ respectively. The weights will be faithful as now the operators are nonsingular. Because $h$ and $k$ have orthogonal supports it also follows easily that $h_{1}$ and $k_{1}$ will commute. Then $\psi$ will be invariant for the modular automorphisms of $\varphi$ given by $\sigma_{t}(x)=h_{1}^{2 i t} x h_{1}^{-2 i t}$ when $x \in B(H)$ and $t \in \mathbf{R}$. We also get $\left\|h_{1} \xi\right\|^{2}=\|h \xi\|^{2}+\|\xi\|^{2}=\|k \xi\|^{2}+\|\xi\|^{2}=\left\|k_{1} \xi\right\|^{2}$ for any $\xi \in D_{0}$ and so if as before $M_{0}$ is the algebra spanned by the operators $\xi \otimes \eta$ with $\xi, \eta \in D_{0}$ then $\psi(x)=\varphi(x)$ for all $x \in M_{0}$.

\section{REFERENCES}

1. U. Haagerup, Operator valued weights on von Neumann algebras. I, J. Funct. Anal. 32 (1979), 175-206.

2. G. K. Pedersen and M. Takesaki, The Radon-Nikodym theorem for von Neumann algebras, Acta Math. 130 (1973), 53-87.

3. A. Van Daele, On pairs of closed operators, preprint (1981).

DEPARTMENT OF MATHEMATICS, UNIVERSITY OF LEUVEN, CELESTIJNENLAAN 200 B, B-3030 HEVERLEE, BELGIUM 